\title{
Das richtige Konzept finden
}

\author{
Peter Michell-Auli
}

\begin{abstract}
Als Management-Instrument ist das Konzept der Balanced Scorecard etabliert. Mit ihr als Denkgerüst ist es auch in der Sozialwirtschaft leichter, alltagstaugliche Strategien zu entwickeln, die Wettbewerbsfähigkeit ermöglichen.
\end{abstract}

Der Markt für soziale Dienstleistungen ist dadurch gekennzeichnet, dass der wirtschaftliche Druck auf die Anbieter seit Jahren zunimmt und dass sie vor der Herausforderung stehen, das Dienstleistungsangebot in einigen Bereichen erheblich umstrukturieren zu müssen. Ebenso ist in der Praxis eine Intensivierung des Wettbewerbs zu bemerken. Die konkurrierenden Unternehmen operieren oftmals mit ähnlichen Strategien. In diesem Umfeld wird die Strategieumsetzung immer wichtiger, um im Markt bestehen zu können.

Bei der Strategieumsetzung und beim Strategiecontrolling kann die Balanced Scorecard mit ihrem syste-

Dr. Peter Michell-Auli hat Volkswirtschaftslehre studiert und an der Universität Köln promoviert. In seinen bisherigen beruflichen Stationen - am Lehrstuhl für Genossenschaftswesen und Sozialpolitik, in der Wirtschaftsprüfung und beim AOK-Bundesverband - hat er sich mit dem Markt für soziale Dienstleistungen beschäftigt. In seiner jetzigen Funktion als Referent für strategische Unternehmensplanung beim Vorstandsvorsitzenden in den Zieglerschen Anstalten betreut er als interner Projektleiter die Einführung der Balanced Scorecard.

E-Mail michellaulip@zieglerischeanstalten.de matischen Ansatz einer Verknüpfung von strategischem Ziel, zugehörigen Messgrößen sowie Zielwerten und den strategischen Aktionen Entscheidendes leisten. Counterpart der Balanced Scorecard ist die »Strategy Map«, in der eine Visualisierung der angestrebten Wertschöpfung durch die Organisation erfolgt. Hierzu werden die Perspektiven in eine Abfolge gebracht, um dann die entscheidenden, vom Management angestrebten Ursachen-Wirkungsbeziehungen zwischen den einzelnen strategischen Zielen deutlich zu machen. Durch die Wahl und die Abfolge der Perspektiven - die sich auch in der Balanced Scorecard wiederfindet und als Architektur bezeichnet wird - wird ein erstes Raster und ein Grundverständnis der angestrebten Wertschöpfung wiedergegeben.

\section{Welche Balanced Scorecard-Archi- tektur zu einem sozialen Unter- nehmen passt}

Die sozialen Unternehmen, die sich im Wesentlichen über Leistungsentgelte finanzieren, stehen - anderes als reine Wohltätigkeitsorganisationen oder öffentliche Verwaltungen - in einem sich verstärkenden Wettbewerb. In diesem wird es immer mehr darauf ankommen, Kundenbedürfnisse zu befriedigen, da nur dies das finanzielle Überleben der Organisation sichert.

Vor dem Hintergrund dieser Marktlogik, die es zu Zeiten des Selbstkostendeckungsprinzips so nicht gab, ist die folgende lineare Perspektivenabfolge sinnvoll: Die Ziele der Lern- und
Entwicklungsperspektive verbessern die internen Prozesse, verbesserte interne Prozesse treiben den Erfolg bei den Kunden an. Der Erfolg bei den Kunden stellt wiederum ein zentrales Element für die Sicherung der angestrebten finanziellen Ergebnisse dar.

Dies hat zur Folge, dass der Erörterung der Architektur einer Balanced Scorecard eines sozialen Unternehmens dem Modell für privat (gewerbliche) Unternehmen entspricht. Entscheidend ist jedoch, dass zwar in beiden Modellen die Finanzperspektive oben steht, die notwendige Interpretation aber doch unterschiedlich ist. Bei der gewerblichen Unternehmung werden in der Finanzperspektive strategische Ziele gebildet, die den Weg hin zu einer optimierten finanziellen Situation weisen. Bei den sozialen Unternehmen geht es in der Finanzperspektive darum, die Überlebensfähigkeit der Organisation zu sichern. Leitende Fragestellung für die Bildung von strategischen Zielen könnte sein: Welche Zielsetzung leitet sich aus der Notwendigkeit ab, unseren finanziellen Spielraum zu erhalten?

Es gibt manchmal einen Disput darüber, ob tatsächlich eine entsprechende Interpretation in der Logik der Balanced Scorecard möglich ist und nicht die oberste Perspektive zu maximieren ist. In der Balanced Scorecard ist man spätestens über die Bildung von Zielwerten in der Lage die Ziele klar zu beschreiben. Also nicht, weil die finanziellen Ziele im Rahmen der unterliegenden Marktlogik ganz oben stehen, heißt dies, dass sie maximiert werden. Eine entsprechende Relativierung kann bereits durch Zielformulierungen - beispielsweise »Ertragskraft erhalten, die Weiterentwicklung ermöglicht « — deutlich gemacht werden.

Wie der altruistische Auftrag dargestellt werden kann

In Abgrenzung zu privat-gewerblichen Unternehmen müssen die sozialen Unternehmen in der Lage sein, die Verfolgung ihres Auftrages in die Balanced Scorecard integrieren zu können. Denn aus der Profilierung des altruistischen Auftrages kann ein Wettbewerbsvorteil gegenüber den gewerblichen Anbietern resultieren. Betrachtet man den altruistischen Auf- 
trag näher, so umfasst dieser sowohl die besondere Motivation zur originären Leistungserstellung, beispielsweise die Altenpflege als auch die Bereitstellung eines Zusatzangebotes.

Für ein diakonisches Unternehmen können beispielsweise folgende strategischen Ziele bestehen, die, abgese- hen von der Leistungserstellung, das "Spezifische « des altruistischen Auftrages ausmachen:

- »Christlichen Glauben erlebbar machen « könnte intendiert sein, dem Kunden den diakonischen Mehrwert unter anderem durch die Schaffung von geistlichen Angeboten $\mathrm{zu}$ vermitteln
- mit dem Ziel »Vernetzung mit Kirchengemeinden ausbauen « könnte eine systematische Vernetzung mit den Kirchengemeinden angestrebt werden

- und mit dem Ziel »christlichen Glauben aktiv leben und erleben « könnte die Stärkung der diakonischen Dienstgemeinschaft ange-

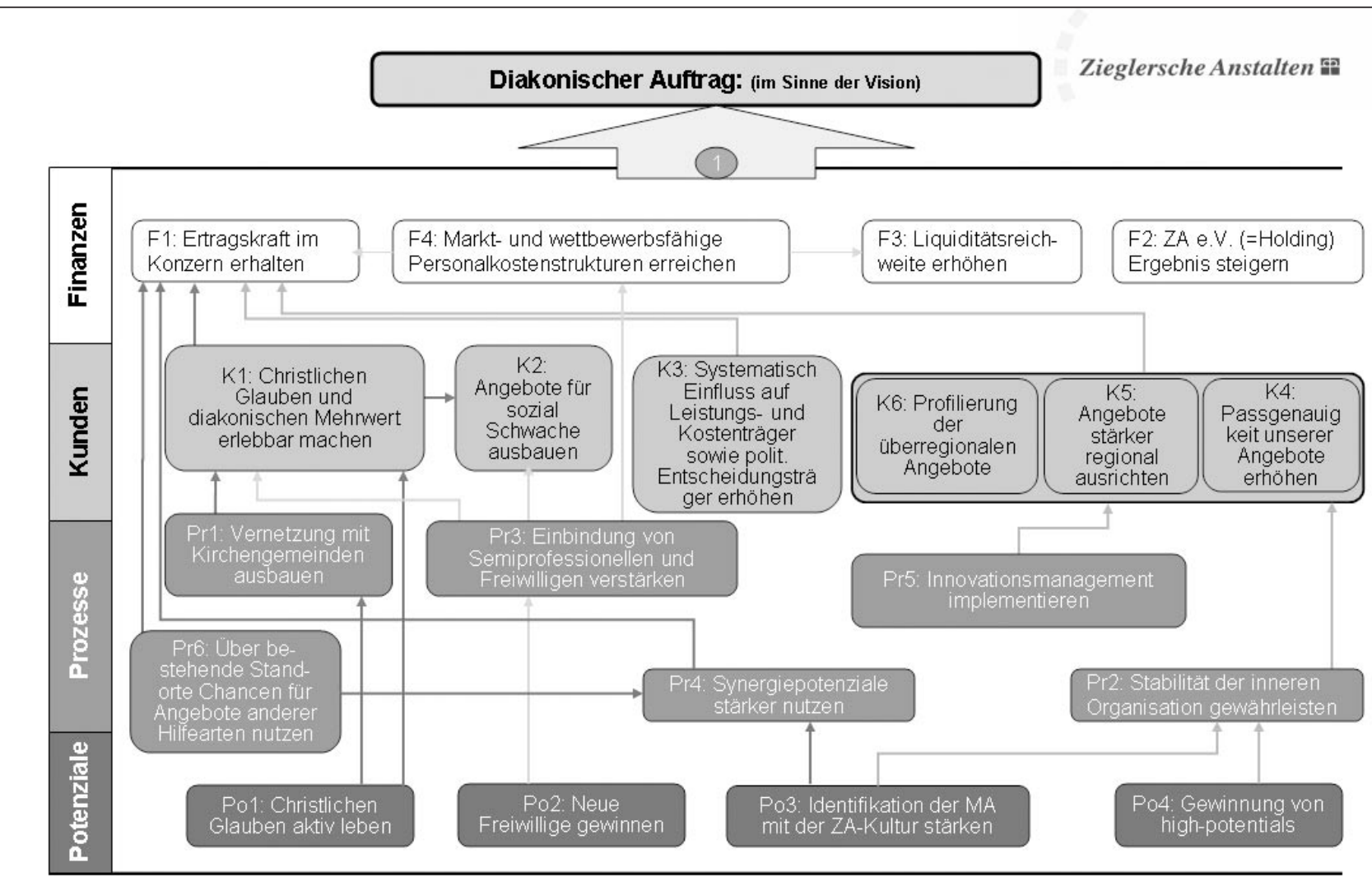

\section{Beispiel Zieglersche Anstalten in Baden-Württemberg}

Vor dem Hintergrund der hier beschriebenen Erwägungen haben sich die Zieglerschen Anstalten für eine eigene Balanced Scorecard entschieden. Die Zieglerschen Anstalten e. V. präsentieren sich als modernes diakonisches Sozialunternehmen mit rund 2.300 Beschäftigten an mehr als 30 Standorten in Baden-Württemberg. Organisiert sind die Zieglerschen Anstalten als Holding mit insgesamt sechs rechtlich selbstständigen Gesellschaften. Aufgrund der integrativ-zusammenfassenden Sicht der Mangement-Holding ließen sich einige strategische Ziele in der Balanced Scorecard nur allgemein formulieren. Beim Herunterbrechen auf die einzelnen Gesellschaften findet derzeit insbesondere bei diesen Zielen eine Konkretisierung für die jeweilige Organisationseinheit statt.

Dem »Spezifischen« des diakonischen Auftrages wurden folgende strategischen Ziele zugeordnet: christlichen Glauben erlebbar machen, Vernetzung mit Kirchengemeinden ausbauen, christlichen Glauben aktiv leben und erleben, Angebote für sozial Schwache ausbauen. Diese Ziele wurden den Perspektiven »Kunden «, »interne Prozesse« und »Potenziale« (entspricht der Perspektive »Lernen und Entwickeln«) zugeordnet. Dass die gesamte Arbeit der Zieglerschen Anstalten der Erfüllung des diakonischen Auftrages dient, wurde durch einen Pfeil deutlich gemacht. (In dem »Dach « wird der diakonische Auftrag in wenigen Leitsätzen für die Zieglerschen Anstalten dargestellt werden. Auf diese Darstellung wurde in der Abbildung verzichtet.)

Die gewählte Perspektivenabfolge — erst Finanzen und dann Kunden — drückt für die Zieglerschen Anstalten die bewusste Notwendigkeit aus, dass die bisherigen Leistungsempfänger zu »Kunden « werden und dass die sozialen Dienstleistungen konsequent an deren Bedürfnissen ausgerichtet werden müssen. Diese Ausrichtung wird dabei als Marktnotwendigkeit verstanden, deren Erfüllung die Voraussetzung bildet, um im Wettbewerb bestehen zu können und damit über den Erhalt des finanziellen Spielraums den diakonische Auftrag fortgesetzt erfüllen zu können. 
strebt werden, etwa durch die Schaffung von Sabbatmonaten.

Diese strategischen Ziele können in das alte Perspektivenraster integriert werden, da es nach wie vor um die Erstellung von Dienstleistungen geht. So könnten die genannten Ziele ohne Probleme den Perspektiven »Kunden «, »innere Prozesse « und »Lernen und Entwickeln« zugeordnet werden. Alternativ könnten die das Spezifische des altruistischen Auftrages betreffenden strategischen Ziele in einer eigenen Perspektive zusammengefasst werden. Im obigen Beispiel könnten die strategischen Ziele der Perspektive »diakonischer Auftrag « zugeordnet werden. Allerdings ergibt sich hier ein gewichtiger Nachteil: Die oben erörterte Logik der Perspektivenabfolge wird zerstört.

Würde man hier aufhören, würde die Balanced Scorecard mit der Finanzperspektive enden. Hier ergeben sich Darstellungsprobleme nach innen und außen beispielsweise gegenüber Kosten- und Leistungsträgern sowie gegenüber Mitarbeitern, da die Praxis zeigt, dass eine oben stehende Finanzperspektive - trotz der obigen Ausführungen — die Vorstellung »Gewinnmaximierung « weckt. Dieses Argument sollte nicht dazu führen, die Finanzperspektive unterhalb der anderen Perspektiven einzuordnen, da es wichtig ist die Marktlogik, der man als Organisation ausgesetzt ist, auch zu kommunizieren. Außerdem macht eine Abbildung der Marktlogik deshalb Sinn, da dieses Denken sich im gesamten Strategieprozess niederschlägt und damit Auswirkungen beispielsweise auf die Strategieanalyse und Strategieentwicklung haben kann.

Allerdings sollte der Eindruck, ein »Gewinnmaximierer « zu sein, vermieden werden, da auch über die Strategiekommunikation mit der Balanced Scorecard das Ziel erreicht werden soll, dass die Strategie »Everyone`s Everyday Job « ist. Wenn daher die Gedankenwelt der Mehrzahl der Mitarbeiter nicht erfasst wird die auch aus altruistischen Motivationen in einem sozialen Unternehmen arbeiten - ist dies durchaus ein ernst zu nehmendes Problem. Auch bei Kosten- und Leistungsträgern wäre bei Pflegesatzverhandlungen der Eindruck nicht hilfreich, dass das Unternehmen einseitig die finanziellen Erträge nach oben schrauben will.
Um dieser Situation auszuweichen, bieten sich zwei Alternativen. An die Spitze der Balanced Scorecard könnte ein übergeordnetes gesellschaftliches Ziel gestellt werden wie beispielsweise die Armutsbekämpfung oder die Verbesserung der Umwelt. Die in der Balanced Scorecard festgelegten Ziele werden dann so gewählt, dass sie zur Erfüllung dieses übergeordneten Ziels beitragen. Die andere Variante besteht darin, ein Dach über die Balanced Scorecard zu setzen, in dem verbal der zugrunde liegende Auftrag der Organisation erläutert wird. In der Strategy Map könnte dann beispielsweise deutlich gemacht werden, dass alle Perspektiven gleicher- maßen zur Erfüllung dieses Auftrages beitragen.

\section{Was soziale Organisationen tun können}

Soziale Unternehmen sollten kein Modell einfach übernehmen. Das Konzept der Balanced Scorecard ist anpassungsfähig, was die Darstellung und Verfolgung der unternehmensindividuellen strategischen Ziele angeht. Die Möglichkeiten der Architektur der Balanced Scorecard sollten im Vorfeld ausreichend erörtert werden, um die Struktur zu finden, die am besten zur eigenen Organisation passt.

\section{Literatur}

BFS Service GmbH: Konzeptheft Balanced Scorecard, Köln 2004

Gaiser, Bernd und Wunder, Thomas: Strategy Maps und Strategieprozess - Einsatzmöglichkeiten, Nutzen, Erfahrungen in: Controlling, Heft 8/9, August/September 2004, S. 457-463.

Horvath \& Partners (Hg.): Balanced Scorecard umsetzen, 3. Auflage, Stuttgart 2004.

Kaplan, Robert S. und Norton, David P.: The Balanced Scorecard - Measures That Drive Performance, in: Harvard Business Review, 1992, Vol. 70, 1, S. 71-79.

Kaplan, Robert S. und Norton, David P.: Die strategie-fokussierte Organisation. Führen mit der Balanced Scorecard, Stuttgart 2001, S. 193-208.

Kaplan, Robert S. und Norton, David P.: Strategy Maps. Der Weg von immateriellen Werten zum materiellen Erfolg, Stuttgart 2004, S. 7-8.

Matysiak, Stefan: Ambulante Assistenz statt Rundum-Betreuung, in epd sozial, Nr.19, 13. Mai 2005.

Michell-Auli, Peter: Chance für den stationären Pflegemarkt: Der Heimvergleich zur Etablierung leistungsgerechter Vergütungssätze, in Gesundheits- und Sozialpolitik, Heft 3-4, 57. Jahrgang.

Horváth \& Partners: Ergebnisbericht: Highlights der Balanced Scorecard Studie 2005, Stuttgart, 2005.

Ottnad, Adrian; Wahl, Stefanie und Miegel, Meinhard: Zwischen Markt und Mildtätigkeit: Die Bedeutung der Freien Wohlfahrtspflege für Gesellschaft, Wirtschaft und Beschäftigung, 2. Auflage, München 2000 .

Stoll, Bettina: Balanced Scorecard für soziale Organisationen, Regensburg 2003.

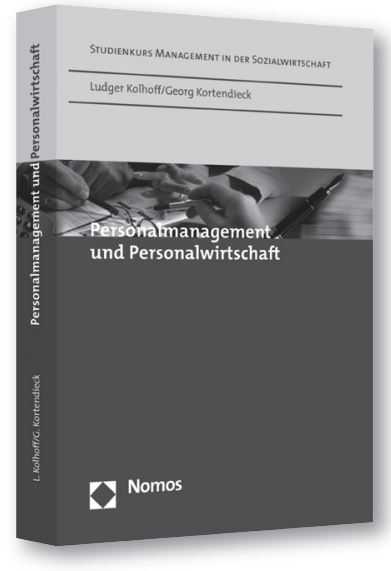

\section{Personalmanagement und Personalwirtschaft}

Von Prof. Dr. phil. Ludger Kolhoff und Prof. Dr. rer. pol. Georg Kortendieck

2006, 176 S., brosch., 19,80€, ISBN 3-8329-1633-4 (Studienkurs Management in der Sozialwirtschaft)

In diesem Band erhalten Studierende und Praktiker einen praxis- wie theoriegeleiteten Überblick über die wichtigsten Bereiche des Personalmanagements und der Personalwirtschaft.

\section{Xomos}

